\title{
Determination of $U_{A}(1)$ restoration from meson
} screening masses by using the entanglement PNJL model: Toward chiral regime

\author{
Masahiro Ishii* \\ Department of Physics, Graduate School of Sciences, Kyushu University, Fukuoka 812-8581, \\ Japan \\ E-mail: ishiiephys.kyushu-u.ac.jp

\section{Junichi Takahashi} \\ Department of Physics, Graduate School of Sciences, Kyushu University, Fukuoka 812-8581, \\ Japan \\ E-mail: takahashiephys.kyushu-u.ac.jp

\section{Hiroaki Kouno} \\ Department of Physics, Saga University, Saga 840-8502, Japan \\ E-mail: kounohdcc.saga-u.ac.ip

\section{Masanobu Yahiro} \\ Department of Physics, Graduate School of Sciences, Kyushu University, Fukuoka 812-8581, \\ Japan \\ E-mail: vahirodphys.kyushu-u.ac.jp
}

We determine temperature $(T)$ dependence of $U_{\mathrm{A}}(1)$ restoration from meson screening masses calculated with $2+1$ flavor lattice QCD, using Polyakov-loop extended Nambu-Jona-Lasinio (PNJL) model with entanglement vertex. The entanglement PNJL (EPNJL) model exhibits the $U_{\mathrm{A}}(1)$ anomaly through the Kobayashi-Maskawa-'t Hooft (KMT) interaction. $T$ dependence of KMT interaction strength is determined from the difference between pion and $a_{0}$ meson screening masses. The strength is strongly suppressed around the pseudocritical temperature of chiral transition. Using this $T$-dependent KMT interaction, we draw the Columbia plot near the physical point. In the light-quark chiral-limit with the strange quark mass fixed at the physical value, the chiral transition becomes the second order. This indicates that there exists a tricritical point. Hence the location is estimated.

The 33rd International Symposium on Lattice Field Theory

14 - 18 July 2015

Kobe International Conference Center, Kobe, Japan

\footnotetext{
* Speaker.
} 


\section{Introduction}

In the Quantum Chromodynamics (QCD) vacuum, $U_{\mathrm{A}}(1)$ symmetry is explicitly broken by the $U_{\mathrm{A}}(1)$ anomaly through the topologically nontrivial gauge configurations. For high temperature $(T)$, the instanton density $d n_{\text {inst }}(T)$ is suppressed by the Debye-type screening [四]:

$$
d n_{\text {inst }}(T) \sim d n_{\text {inst }}(0) \exp \left[-\pi^{2} \rho^{2} T^{2}\left(\frac{2}{3} N_{c}+\frac{1}{3} N_{f}\right)\right],
$$

where $N_{c}\left(N_{f}\right)$ means number of colors (flavors) and $\rho$ means instanton radius. The suppresion suggests that $U_{\mathrm{A}}(1)$ symmetry is effectively restored at high temperature. The restoration of $U_{\mathrm{A}}(1)$ symmetry is related with the order of the chiral phase transition in 2-flavor QCD at zero light-quark mass. In Ref. [Z], it is suggested that the order may be second order with 3d Izing $O(4)$ universality class if the effective restoration is not completed at $T=T_{c}$, where $T_{c}$ is transition temperature for chiral phase transition. When $U_{\mathrm{A}}(1)$ symmetry is restored completely at $T=T_{c}$, the chiral transition becomes the first order [回]. Recently, however, it was pointed out in Ref. [B] that the second order is still possible. In this case, the universality class is not $O(4)$ but $U(2)_{\mathrm{L}} \times U(2)_{\mathrm{R}}$. There are many lattice QCD (LQCD) simulations and effective model analyses made so far to clarify the order and its universality class in the two-flavor chiral limit and the light-quark chiral limit where light-quark mass vanishes with strange-quark mass fixed at the physical value, but these are still controversial.

In this talk, we incorporate the effective restoration of $U_{\mathrm{A}}(1)$ symmetry in entantlement Polyakovloop extended Nambu-Jona-Lasinio (EPNJL) model by introducing a temperature-dependent strength $K(T)$ to the Kobayashi-Maskawa-' $t$ Hooft (KMT) determinant interaction. $T$ dependence of $K(T)$ is well determined from the results of state-of-the-art 2+1-flavor lattice QCD simulations on pion and $a_{0}$-meson screening masses. Using the EPNJL model, we draw the Columbia plot near the physical point and determine the order of chiral transition in the light-quark chiral limit with $m_{s}$ fixed at the physical value.

\section{Model setting}

\subsection{EPNJL model}

We start with the $2+1$ flavor EPNJL model [四]. The Lagrangian density is

$$
\begin{aligned}
\mathscr{L} & =\bar{\psi}\left(i \gamma_{\mu} D^{\mu}-\hat{m}_{0}\right) \psi+G_{\mathrm{s}}(\Phi) \sum_{a=0}^{8}\left[\left(\bar{\psi} \lambda_{a} \psi\right)^{2}+\left(\bar{\psi} i \gamma_{5} \lambda_{a} \psi\right)^{2}\right] \\
& -K(T)\left[\operatorname{det}_{f, f^{\prime}} \bar{\psi}_{f}\left(1+\gamma_{5}\right) \psi_{f^{\prime}}+\operatorname{det}_{f, f^{\prime}} \bar{\psi}_{f}\left(1-\gamma_{5}\right) \psi_{f^{\prime}}\right]-\mathscr{U}(\Phi[A], \bar{\Phi}[A], T)
\end{aligned}
$$

with quark fields $\psi=\left(\psi_{u}, \psi_{d}, \psi_{s}\right)^{T}$ and $D^{\mu}=\partial^{\mu}+i A^{\mu}$ with $A^{\mu}=\delta_{0}^{\mu} g\left(A^{0}\right)_{a} t_{a} / 2=-\delta_{0}^{\mu} i g\left(A_{4}\right)_{a} t_{a} / 2$ for the gauge coupling $g$, where the $\lambda_{a}\left(t_{a}\right)$ are the Gell-Mann matrices in flavor (color) space and $\lambda_{0}=\sqrt{2 / 3} \mathbf{I}$ for the unit matrix $\mathbf{I}$ in flavor space. The determinant in (2. I) is taken in flavor space. For the $2+1$ flavor system, the current quark masses $\hat{m}_{0}=\operatorname{diag}\left(m_{u}, m_{d}, m_{s}\right)$ satisfy a relation $m_{s}>m_{l} \equiv m_{u}=m_{d}$. In the EPNJL model, the coupling strength $G_{\mathrm{s}}(\Phi)$ of the scalar-type four-quark interaction depends on the Polyakov loop $\Phi$ and its Hermitian conjugate $\bar{\Phi}$ as

$$
G_{\mathrm{s}}(\Phi)=G_{\mathrm{s}}(0) \times\left[1-\alpha_{1} \Phi \bar{\Phi}\right] .
$$


This entanglement coupling is charge-conjugation and $Z_{3}$ symmetric.

For $T$ dependence of $K(T)$, we assume the following form phenomenologically:

$$
K(T)=\left\{\begin{array}{ll}
K(0) & \left(T<T_{1}\right) \\
K(0) e^{-\left(T-T_{1}\right)^{2} / b^{2}} & \left(T \geq T_{1}\right)
\end{array} .\right.
$$

For high $T$ satisfying $T \gg T_{1}$, the form (ㄹ.3]) is reduced to ([D.

In the EPNJL model, the time component of $A_{\mu}$ is treated as a homogeneous and static background field, which is governed by the Polyakov-loop potential $\mathscr{U}$. In the Polyakov gauge, $\Phi$ and $\bar{\Phi}$ are obtained by

$$
\Phi=\frac{1}{3} \operatorname{tr}_{\mathrm{c}}(L), \quad \bar{\Phi}=\frac{1}{3} \operatorname{tr}_{\mathrm{c}}\left(L^{*}\right)
$$

with $L=\exp \left[i A_{4} / T\right]=\exp \left[i \operatorname{diag}\left(A_{4}^{11}, A_{4}^{22}, A_{4}^{33}\right) / T\right]$ for real variables $A_{4}^{j j}$ satisfying $A_{4}^{11}+A_{4}^{22}+$ $A_{4}^{33}=0$. For zero chemical potential where $\Phi=\bar{\Phi}$, one can set $A_{4}^{33}=0$ and determine the others as $A_{4}^{22}=-A_{4}^{11}=\cos ^{-1}[(3 \Phi-1) / 2]$.

We use the logarithm-type Polyakov-loop potential of Ref. [目] as $\mathscr{U}$, but we refit the parameter $T_{0}$ to $180 \mathrm{MeV}$ in order to reproduce the chiral transition temperature $T_{c}=154 \pm 9 \mathrm{MeV}$ [ [6, 口, (8] and deconfinement transition temperature $T_{c}^{\text {deconf }}=170 \pm 7 \mathrm{MeV}$ [Q] ].

Making the mean field approximation (MFA) to (D.d) and the path integral over quark fields, one can get the thermodynamic potential (per unit volume) as

$$
\begin{array}{r}
\Omega=U_{\mathbf{M}}+\mathscr{U}-2 \sum_{f=u, d, s} \int \frac{d^{3} \mathbf{p}}{(2 \pi)^{3}}\left[3 E_{\mathbf{p}, f}+\frac{1}{\beta} \ln \left[1+3\left(\Phi+\bar{\Phi} e^{\left.-\beta E_{\mathbf{p}, f}\right)} e^{-\beta E_{\mathbf{p}, f}}+e^{-3 \beta E_{\mathbf{p}, f}}\right]\right.\right. \\
+\frac{1}{\beta} \ln \left[1+3\left(\bar{\Phi}+\Phi e^{-\beta E_{\mathbf{p}, f}}\right) e^{-\beta E_{\mathbf{p}, f}}+e^{-3 \beta E_{\mathbf{p}, f}}\right]
\end{array}
$$

with $\beta=1 / T$ and $E_{\mathbf{p}, f}=\sqrt{\mathbf{p}^{2}+M_{f}^{2}}$. The effective quark mass $M_{f}$ is $M_{f}=m_{f}-4 G_{\mathrm{s}}(\Phi) \sigma_{f}+$ $2 K(T) \sigma_{f^{\prime}} \sigma_{f^{\prime \prime}}$ with $f \neq f^{\prime} \neq f^{\prime \prime}$. The mesonic potential $U_{\mathrm{M}}$ is $U_{\mathrm{M}}=2 G_{\mathrm{s}}(\Phi)\left(\sigma_{u}^{2}+\sigma_{d}^{2}+\sigma_{s}^{2}\right)-$ $4 K(T) \sigma_{u} \sigma_{d} \sigma_{s}$. Here, $\sigma_{f}$ means the chiral condensate $\left\langle\bar{\psi}_{f} \psi_{f}\right\rangle$ for flavor $f$. We determine the mean-field variables $\left(X=\sigma_{l}, \sigma_{s}, \Phi, \bar{\Phi}\right)$ from the stationary conditions:

$$
\frac{\partial \Omega}{\partial X}=0
$$

where isospin symmetry is assumed for the light-quark sector, i.e., $\sigma_{l} \equiv \sigma_{u}=\sigma_{d}$.

On the right-hand side of (2.5]), the first term (vacuum term) in the momentum integral diverges. We then use the PV regularization [ए]]. In the scheme, the integral $I\left(M_{f}\right)$ is regularized as

$$
I^{\mathrm{reg}}\left(M_{f}\right)=\sum_{\alpha=0}^{2} C_{\alpha} I\left(M_{f ; \alpha}\right),
$$

where $M_{f ; 0}=M_{f}$ and the $M_{f ; \alpha}(\alpha \geq 1)$ mean masses of auxiliary particles. The parameters $M_{f ; \alpha}$ and $C_{\alpha}$ should satisfy the condition $\sum_{\alpha=0}^{2} C_{\alpha}=\sum_{\alpha=0}^{2} C_{\alpha} M_{f ; \alpha}^{2}=0$. We then assume $\left(C_{0}, C_{1}, C_{2}\right)=$ $(1,1,-2)$ and $\left(M_{f ; 1}^{2}, M_{f ; 2}^{2}\right)=\left(M_{f}^{2}+2 \Lambda^{2}, M_{f}^{2}+\Lambda^{2}\right)$. We keep the parameter $\Lambda$ finite even after the 
subtraction ([2]), since the present model is non-renormalizable. The parameters are taken from Ref. [प]] and they are $m_{l}=6.2 \mathrm{MeV}, m_{s}=175.0 \mathrm{MeV}, G_{\mathrm{s}}(0) \Lambda^{2}=2.35$ and $K(0) \Lambda^{5}=27.8$ for $\Lambda=795 \mathrm{MeV}$. This parameter set reproduces mesonic observables at vacuum, i.e., the pion and kaon decay constants $\left(f_{\pi}=92 \mathrm{MeV}\right.$ and $\left.f_{K}=105 \mathrm{MeV}\right)$ and their masses $\left(M_{\pi}=141 \mathrm{MeV}\right.$ and $\left.M_{K}=512 \mathrm{MeV}\right)$ and the $\eta^{\prime}$-meson mass $\left(M_{\eta^{\prime}}=920 \mathrm{MeV}\right)$. In the present work, we analyze LQCD results of Ref. [[2] for pion and $a_{0}$-meson screening masses. In the LQCD simulation, the pion mass $M_{\pi}(0)$ at vacuum $(T=0)$ is $175 \mathrm{MeV}$ and a bit heavier than the experimental value $138 \mathrm{MeV}$. We then change $m_{l}$ to $9.9 \mathrm{MeV}$ in the EPNJL model in order to reproduce $M_{\pi}(0)=175 \mathrm{MeV}$.

\subsection{Meson screening mass}

We derive the equations for pion and $a_{0}$-meson screening masses, following Ref [[13], [4]]. The current corresponding to a meson of type $\xi$ is

$$
J_{\xi}(x)=\bar{\psi}(x) \Gamma_{\xi} \psi(x)-\left\langle\bar{\psi}(x) \Gamma_{\xi} \psi(x)\right\rangle,
$$

where $\Gamma_{\pi}=i \gamma_{5} \lambda_{3}$ for $\pi$ meson and $\Gamma_{a_{0}}=\lambda_{3}$ for $a_{0}$ meson. We denote the Fourier transform of the mesonic correlation function $\eta_{\xi \xi}(x) \equiv\left\langle 0\left|\mathrm{~T}\left(J_{\xi}(x) J_{\xi}^{\dagger}(0)\right)\right| 0\right\rangle$ by $\chi_{\xi \xi}\left(q_{0}^{2}, \tilde{q}^{2}\right)$ as

$$
\chi \xi \xi\left(q_{0}^{2}, \tilde{q}^{2}\right)=i \int d^{4} x e^{i q \cdot x} \eta_{\xi \xi}(x)
$$

where $\tilde{q}= \pm|\mathbf{q}|$ for $q=\left(q_{0}, \mathbf{q}\right)$ and $\mathrm{T}$ stands for the time-ordered product. Using the random-phase (ring) approximation, one can obtain the Schwinger-Dyson equations for $\xi=\pi, a_{0}$ channels

$$
\chi_{\xi \xi}=\frac{\Pi_{\xi}}{1-2 G_{\xi} \Pi_{\xi}}
$$

with the effective couplings $G_{\pi}$ and $G_{a_{0}}$ defined by

$$
G_{a_{0}}=G_{\mathrm{s}}(\Phi)+\frac{1}{2} K(T) \sigma_{s}, G_{\pi}=G_{\mathrm{s}}(\Phi)-\frac{1}{2} K(T) \sigma_{s},
$$

and the one-loop polarization function $\Pi_{\xi}$ defined by

$$
\Pi_{a_{0}}=4 i\left[I_{1}+I_{2}-\left(q^{2}-4 M^{2}\right) I_{3}\right], \quad \Pi_{\pi}=4 i\left[I_{1}+I_{2}-q^{2} I_{3}\right] .
$$

$M$ is the effective light-quark mass and $M=M_{u}=M_{d}$. At $T=0$, three integrals $I_{1}, I_{2}, I_{3}$ are obtained by

$$
\begin{aligned}
& I_{1}=\int \frac{d^{4} p}{(2 \pi)^{4}} \operatorname{tr}_{\mathrm{c}}\left[\frac{1}{p^{\prime 2}-M}\right], I_{2}=\int \frac{d^{4} p}{(2 \pi)^{4}} \operatorname{tr}_{\mathrm{c}}\left[\frac{1}{\left(p^{\prime}+q\right)^{2}-M^{2}}\right] \\
& I_{3}=\int \frac{d^{4} p}{(2 \pi)^{4}} \operatorname{tr}_{\mathrm{c}}\left[\frac{1}{\left\{\left(p^{\prime}+q\right)^{2}-M^{2}\right\}\left(p^{\prime 2}-M^{2}\right)}\right]
\end{aligned}
$$

with $p^{\prime}=\left(p_{0}+i A_{4}, \mathbf{p}\right)$. $\operatorname{tr}_{\mathrm{c}}$ means the trace in color space. For finite $T$, the corresponding equations are obtained by the replacement

$$
p_{0} \rightarrow i \omega_{n}=i(2 n+1) \pi T, \quad \int \frac{d^{4} p}{(2 \pi)^{4}} \rightarrow i T \sum_{n=-\infty}^{\infty} \int \frac{d^{3} \mathbf{p}}{(2 \pi)^{3}} .
$$


Meson screening mass is defined by the exponential damping of the meson propagator $\eta_{\xi \xi}(r)$ in the long distance limit $(r \rightarrow \infty)$ :

$$
M_{\xi, \mathrm{scr}}=-\lim _{r \rightarrow \infty} \frac{d \ln \eta_{\xi \xi}(r)}{d r}
$$

where $\eta_{\xi \xi}(r)$ is obtained by the Fourier transform of $\chi_{\xi \xi}\left(0, \tilde{q}^{2}\right)$ from the momentum $\tilde{q}$ space to the coordinate space $r$ :

$$
\eta_{\xi \xi}(r)=\frac{1}{4 \pi^{2} i r} \int_{-\infty}^{\infty} d \tilde{q} \tilde{q} \chi_{\xi \xi}\left(0, \tilde{q}^{2}\right) e^{i \tilde{q} r}
$$

It is not easy to make this Fourier transformation particularly at large $r$ due to the highly oscilating function $e^{i \tilde{q} r}$. In order to avoid this problem, one can consider the Fourier transformation as a contour integral in the complex $\tilde{q}$ plane by using the Cauchy's integral theorem. However, it is reported in Ref. [ㄷ]] that $\chi_{\xi \xi}\left(0, \tilde{q}^{2}\right)$ has logarithmic cuts in the vicinity of the real $\tilde{q}$ axis and heavy numerical calculations are necessary for evaluating the cut effects. In our previous work [14]], we showed that these logarithmic cuts are unphysical and removable. If we make the $\mathbf{p}$ integral before taking the Matsubara summation $\sum_{n}$ in $\left(\left[2 . \mathbb{L}^{2}\right)\right.$, we can express $I_{3}^{\text {reg }}\left(0, \tilde{q}^{2}\right)$ as an infinite series of analytic functions:

$$
I_{3}^{\mathrm{reg}}\left(0, \tilde{q}^{2}\right)=\frac{i T}{4 \pi \tilde{q}} \sum_{j, n, \alpha} C_{\alpha} \sin ^{-1}\left(\frac{\frac{\tilde{q}}{2}}{\sqrt{\frac{\tilde{q}^{2}}{4}+M_{j, n, \alpha}^{2}}}\right)
$$

with

$$
M_{j, n, \alpha}(T)=\sqrt{M_{\alpha}^{2}+\left\{(2 n+1) \pi T+A_{4}^{j j}\right\}^{2}},
$$

where $M_{\alpha}=M_{u ; \alpha}=M_{d ; \alpha}$. Each term of $I_{3}^{\mathrm{reg}}\left(0, \tilde{q}^{2}\right)$ has two physical cuts on the imaginary axis, one is an upward vertical line starting from $\tilde{q}=2 i M_{j, n, \alpha}$ and the other is a downward vertical line from $\tilde{q}=-2 i M_{j, n, \alpha}$. There are two lowest branch points $\tilde{q}=2 i M_{j=1, n=0, \alpha=0}=2 i M_{j=2, n=-1, \alpha=0}$. We call them "threshold mass" in the sense that they come from the quark- antiquark continuum state.

We can obtain the meson screening mass $M_{\xi \text {,scr }}$ as a pole of $\chi_{\xi \xi}\left(0, \tilde{q}^{2}\right)$,

$$
\left.\left[1-2 G_{\xi} \Pi_{\xi}\left(0, \tilde{q}^{2}\right)\right]\right|_{\tilde{q}=i M_{\xi, \mathrm{scr}}}=0 .
$$

If the pole at $\tilde{q}=i M_{\xi, \text { scr }}$ is well isolated from the cut, i.e., $M_{\xi, \text { scr }}<2 M_{j=1, n=0, \alpha=0}$, one can determine the screening mass from the pole location without making the $\tilde{q}$ integral.

\section{Numerical Results}

The EPNJL model has three adjustable parameters, $\alpha_{1}$ in the entanglement coupling $G_{\mathrm{s}}(\Phi)$ and $b$ and $T_{1}$ in the KMT interaction $K(T)$. These parameters can be clearly determined from $2+1$ flavor LQCD data [12] for pion and $a_{0}$-meson screening masses, $M_{\pi, \mathrm{scr}}$ and $M_{a_{0}, \mathrm{scr}}$ as shown below.

Figure 1 shows $T$ dependence of $M_{\pi, \mathrm{scr}}, M_{a_{0}, \mathrm{scr}}$. The EPNJL results for $M_{\pi, \mathrm{scr}}\left(M_{a_{0}, \mathrm{scr}}\right)$ are represented by solid (dotted) line and LQCD ones are plotted with closed squares (open circles). Best fitting is obtained, when $\alpha_{1}=1.0, T_{1}=0.79 T_{c}=121 \mathrm{MeV}$ and $b=0.23 T_{c}=36 \mathrm{MeV}$. The 


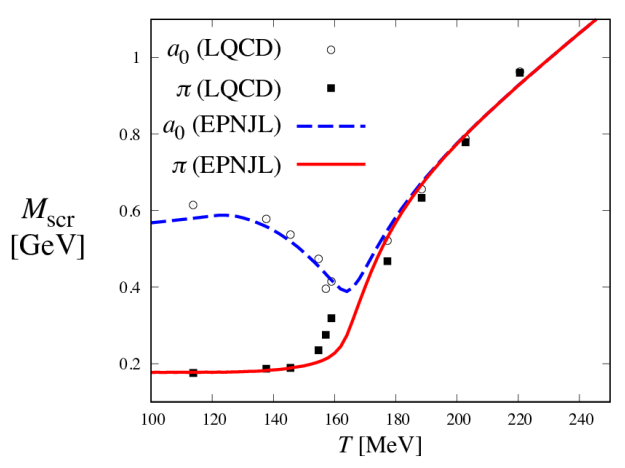

Fig. 1: $T$ dependence of $M_{\pi, \text { scr }}$ and $M_{a_{0}, \text { scr. }}$. The solid (dotted) line denotes $M_{\pi, \text { scr }}\left(M_{a_{0}, \text { scr }}\right)$ calculated by the EPNJL model. LQCD data are taken from Ref. [ए]]; closed squares (open circles) correspond to the $2+1$ flavor data for $M_{\pi, \mathrm{scr}}\left(M_{a_{0}, \mathrm{scr}}\right)$. LQCD data are rescaled by the factor $154 / 196$.

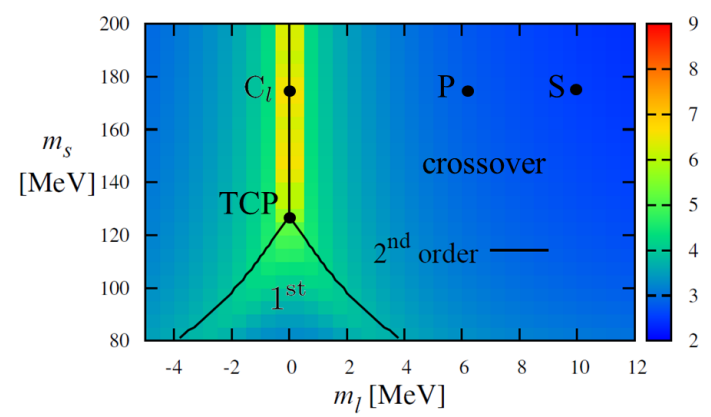

Fig. 2: Order of chiral transition near physical point in the $m_{l}-m_{s}$ plane. The value of $\log \left[\chi_{l l}\left(T_{c}\right)\right]$ is shown by a change in hue. Simulation point, physical point, light-quark chirallimit point and tricritical point are denoted by $\mathrm{S}$, $\mathrm{P}, \mathrm{C}_{l}$ and TCP. The solid lines stand for secondorder chiral transitions.

parameters thus obtained lead to $K\left(T_{c}\right) / K(0)=0.434$ and it indicates the rapid suppression of $K(T)$ in the vicinity of $T_{c}$, but $U_{\mathrm{A}}(1)$ symmetry is not completely restored at $T_{c}$. Here, we rescale the LQCD results of [12] with multiplying them by the factor $154 / 196$ to reproduce $T_{c}=154 \pm 9$ $\mathrm{MeV}$. This is because $T_{c}$ in the simulations [12] is about $196 \mathrm{MeV}$, although it becomes $T_{c}=154 \pm 9$ $\mathrm{MeV}$ in finer LQCD simulations [ [ , [, [] ] close to the continuum limit.

Using the $T$-dependent KMT interaction, we draw the Columbia plot near the physical point in $m_{l}-m_{s}$ plane, as shown in Fig. $\square$. The $\mathrm{S}$ point represents the location of $\left(m_{l}, m_{s}\right)$ for the LQCD simulation [[2] and it is located at $\left(m_{l}, m_{s}\right)=(9.9[\mathrm{MeV}], 175[\mathrm{MeV}])$. S-point is close to lightquark chiral limit point $\left(C_{l}\right)$, therefore, we can extrapolate the LQCD results [ए2] from S-point to $C_{l}$-point by using the EPNJL model. Varying both $m_{l}$ and $m_{s}$, we determine the order of chiral transition from the divergence of $T$ dependence of chiral susceptibility $\chi_{l l}(T)$ and the discontinuity of chiral condensate. The value of $\log \left[\chi_{l l}\left(T_{c}\right)\right]$ is denoted by a change in hue. In the light-quark chiral-limit with the strange quark mass $\left(m_{s}\right)$ fixed at the physical value $m_{s}=m_{s}^{\text {phys }}=175 \mathrm{MeV}$, the chiral transition becomes the second order in the mean field approximation. The second-order chiral transitions (solid lines) meet at $\left(m_{l}^{\text {tric }}, m_{s}^{\text {tric }}\right) \approx\left(0,0.726 m_{s}^{\text {phys }}\right)=(0[\mathrm{MeV}], 127[\mathrm{MeV}])$. This is a tricritical point (TCP) of chiral phase transition.

\section{Summary}

In summary, we incorporated the effective restoration of $U_{\mathrm{A}}(1)$ symmetry in the $2+1$ flavor EPNJL model by introducing a $T$-dependent coupling strength $K(T)$ to the KMT interaction. The $T$ dependence was well determined from state-of-the-art $2+1$ flavor LQCD data on pion and $a_{0^{-}}$ meson screening masses. The strength $K(T)$ thus obtained is suppressed in the vicinity of the 
pseudocritical temperature of chiral transition. However, $U_{\mathrm{A}}(1)$ symmetry breaking still remains at $T_{c}$. Using the EPNJL model with the present parameter set, we showed that, at least in the mean field level, the order of chiral transition is second order at the light-quark chiral-limit point of $m_{l}=0$ and $m_{s}=175 \mathrm{MeV}$ (the physical value). This result indicates that there exists a tricritical point near the light-quark chiral-limit point in the $m_{l}-m_{s}$ plane. We then estimated the location of the tricritical point as $\left(m_{l}^{\text {tric }}, m_{s}^{\text {tric }}\right) \approx\left(0,0.726 m_{s}^{\text {phys }}\right)=(0[\mathrm{MeV}], 127[\mathrm{MeV}])$.

\section{Acknowledgments}

M. I, J. T., H. K., and M. Y. are supported by Grant-in-Aid for Scientific Research ( No. 273944, No. 25-3944, No. 26400279 and No. 26400278) from the Japan Society for the Promotion of Science (JSPS).

\section{References}

[1] R. D. Pisarski, and L. G. Yaffe, Phys. Lett. B 97,

[2] R. D. Pisarski, and F. Wilczek, Phys. Rev. D 29, 338(R) (1984). 110 (1980).

[3] A. Pelissetto, and E. Vicari, Phys. Rev. D 88, 105018 (2013).

[4] T. Sasaki, Y. Sakai, H. Kouno, and M. Yahiro, Phys. Rev. D 84, 091901(R) (2011).

[5] S. Rößner, C. Ratti, and W. Weise, Phys. Rev. D 75, 034007 (2007).

[6] S. Borsanyi et al., J. High Energy Phys. 09 (2010) 073.

[7] A. Bazavov, T. Bhattacharya, M. Cheng, C. DeTar, H. -T. Ding, S. Gottlieb, R. Gupta, and P. Hegde et al., Phys. Rev. D 85, 054503 (2012).

[8] K. Kanaya, [arXiv: 1012.4235]; [arXiv: 1012.4247].

[9] Y. Aoki, S. Borsányi, S. Dürr, Z. Fodor, S. D. Katz, S. Krieg, and K. K. Szabo, J. High Energy Phys. 06 (2009) 088 [arXiv:0903.4155].

[10] W. Pauli, and F. Villars, Rev. Mod. Phys. 21, 434 (1949).

[11] V. Bernard, and D. Vautherin, Phys. Rev. D 40, 1615 (1989).

[12] M. Cheng, S. Datta, A. Francis, J. van der Heide, C. Jung, O. Kaczmarek, F. Karsch, and E. Laermann et al., Eur. Rhys. J. C 71, 1564 (2011).

[13] S. P. Klevansky Rev. Mod. Phys. 64, 649 (1992); T. Hatsuda, and T. Kunihiro Phys. Rep. 247, 221 (1994); M. Buballa Phys. Rep. 407, 205 (2005).

[14] M. Ishii, T. Sasaki, K. Kashiwa, H. Kouno, and M. Yahiro, Phys. Rev. D 89, 071901(R) (2014).

[15] W. Florkowski, Acta Phys. Pol. B 28, 2079 (1997) . 